\title{
MiR-155 and MiR-665 role as potential non-invasive biomarkers for hepatocellular carcinoma in Egyptian patients with chronic hepatitis $\mathrm{C}$ virus infection
}

Amal Ahmed Mohamed ${ }^{1}$, Abdellah Abosrie Ali Omar'2,

Rehab R. EL-Awady ${ }^{3}$, Sally Mohamed Aboelsayed Hassan ${ }^{3}$, Waleed Mohamed Soliman Eitah ${ }^{4}$, Rehab Ahmed ${ }^{5}$, Amir khater ${ }^{5}$, Omnia Mohamed Saad Tantawi ${ }^{6}$, Ahmed Abdelhafeez Mohamed ${ }^{7}$

${ }^{1}$ Biochemistry and Molecular Biology Department, National Hepatology and Tropical Medicine Research institute, Cairo, Egypt;

${ }^{2}$ Medical Biochemistry Department, Faculty of Medicine (Boys), Al-Azhar University Cairo, Egypt; ${ }^{3}$ Biochemistry Department, Faculty of Pharmacy (Girls), AL-Azhar University Cairo, Egypt; ${ }^{4}$ Gastroenterology and Hepatology Department, Theodor Bilharz Research Institute, Cairo, Egypt;

${ }^{5}$ Tropical Department, National Hepatology and Tropical Medicine Research Institute, Cairo, Egypt; ${ }^{6}$ Endemic Diseases Department, Kasraliny, Cairo University, Cairo, Egypt; ${ }^{7}$ Clinical Oncology Department, Kasralainy, Cairo University, Cairo, Egypt

Address for Correspondence: Dr. Ahmed Abdelhafeez Mohamed, Clinical Oncology Department,

Kasralainy, Cairo University, Cairo, Egypt

E-mail: hbaboda@kasralainy.edu.eg

\begin{tabular}{|l|}
\hline Access this article online \\
\hline Website: \\
www.intern-med.com \\
\hline DOI: \\
10.2478/jtim-2020-0006 \\
\hline Quick Response Code: \\
\hline \\
\\
\end{tabular}

\section{ABSTRACT}

Background and Objectives: Hepatocellular carcinoma (HCC) is the fourth leading cause of cancer associated death globally. Serum micro RNAs are full of potential as noninvasive biomarkers. Here, we aim to assess the performance of serum MicroRNA-155 and MicroRNA-665 as diagnostic biomarker for HCC comparing to AFP. Methods: Serum samples were collected from 200 subjects $(40$ healthy control, 80 chronic hepatitis $C$ patients with cirrhosis and without HCC (LC) and $80 \mathrm{HCC}$ patients currently infected by hepatitis C infection and didn't start the treatment). The HCC patients didn't include alcoholic liver disease, nonalcoholic fatty liver disease nor autoimmune liver disease. MicroRNA-155 and MicroRNA-665 expression were measured by real-time quantitative PCR (RT-qPCR), while AFP level was assessed by ELISA method. Results: Both miR-155 and miR-665 were significantly elevated in HCC group as compared to both control and LC groups. The comparison between LC and HCC patients revealed that the serum level of miR-155 was a significant increase in HCC patients compared to LC patients; however, the serum level of miR-665 didn't show any significant difference between the same two groups. MiR-665 expression level showed a direct correlation with tumor size in HCC patients. Conclusions: Using measurement against AFP level in serum, miR-665 is considered a promising serum biomarker for the diagnosis of HCC patients among the LC patients without HCC. MiR-155 didn't provide a better performance than serum AFP as a diagnostic biomarker among the same group. MiR-665 may serve as a good indicator for HCC prognosis.

Key words: hepatocellular carcinoma (HCC), chronic hepatitis with cirrhosis $\mathrm{C}$ and without $\mathrm{HCC}$ (LC), alpha-fetoprotein (AFP), micro RNAs (miRNA)

\section{INTRODUCTION}

Hepatitis $\mathrm{C}$ virus (HCV), is one of the leading causes of chronic liver disease. Hepatocellular carcinoma (HCC) is a major complication associated with $\mathrm{HCV}$ virus infection, with significant mortality and morbidity rates. ${ }^{[1]}$ Egypt holds the highest record of HCV infection globally with almost $15 \%$ of the population. ${ }^{[2]}$
Hepatocellular carcinoma is the most common type of liver cancer and a main cause of tumor-related mortality. According to global cancer statistics from 2012, HCC is the second leading cause of cancer-related deaths in men and the sixth in women, worldwide, and the HCC incidence is still rising globally. ${ }^{[3]}$ The overall 5-year survival rate is very low because of the high aggressiveness of the cancer and the 
limited therapeutic options. The most effective therapies as resection or liver transplantation only work in patients at an early stage of the disease. HCC in most cases occurs in the chronically inflamed and damaged liver. ${ }^{[4]}$

In screening and detection of HCC in high risk patients as chronic hepatitis $\mathrm{C}(\mathrm{CHC})$ patients, the most used method is combination of Ultrasonography and serum level of AFP. The disadvantages of ultrasonography are failure of detection of small tumors, operator dependence and diverse diagnostic accuracy. ${ }^{[5,6]}$ As for the AFP serum level, it has many drawbacks as low sensitivity and specificity, the upregulation in some patients with cirrhosis or/and hepatic inflammation in absence of the tumor and no elevation in $80 \%$ of small tumor. ${ }^{[7,8]}$

Hepatocellular carcinoma development and progression are caused by the accumulation of genetic changes that results in tumor related genes expression: oncogenes, tumor suppressor genes, genes involved in many regulatory pathways, such as cell cycle control, apoptosis and angiogenesis. Expression of thousands of mRNAs can be measured at the same time due to advanced technology; this provides a thorough data for both diagnosis and therapy of HCC. ${ }^{[9]}$

MiRNAs are defined as small non-coding RNA molecules that mediate multiple physiological and pathological processes such as inflammation and cancer. ${ }^{[10]}$ MiRNAs are present in body fluids as serum and plasma, mostly in exosomes or protein-RNA-complexes, which protect them against degradation caused by RNase and other harsh conditions. ${ }^{[11]}$

Extracellular miRNAs seem to be the by-products of dead or dying cells and stay in the extracellular space because of the high stability of the Argonaute2-miRNA complex ${ }^{[12]}$ or the presence of lipoprotein complexes ${ }^{[13]}$ or vesicles, ${ }^{[14]}$ which may preserve stability. Setting aside of the mechanism, miRNAs can be detected and quantified by PCR using specific primers, making them potentially functional biomarkers. ${ }^{[12]}$

Dysregulation of miRNAs expression has been demonstrated in various types of cancer including HCC. ${ }^{[15]}$ In addition, circulating miRNAs have been showed as a promising biomarkers for HCC diagnosis and/or good prognosis. ${ }^{[7]}$

There is a need for new biomarkers that are more disease reflective and more sensitive for identification of HCC patients. Recently, miR-665 was found to be elevated in the tissue of HCC. ${ }^{[16]}$ In addition, miR-155 was linked to both $\mathrm{CHC}$ infection and HCC. ${ }^{[17,18]}$
Based on the previous information, the main aim of this study is to explore the usefulness of two serum miRNAs miR-155 and miR-665 as potential noninvasive biomarkers for the diagnosis of HCC.

\section{PATIENTS AND METHODS}

This study was approved by Department of Gastroenterology and Hepatology, Theodor Bilharz Research Institute. Serum samples were collected from a total number of 200 subjects. All patients were recruited from the Department of Gastroenterology and Hepatology, Theodor Bilharz Research Institute during the period from October 2017 to November 2018. Forty healthy volunteers were involved in the current study as a control group. Both patients and volunteers signed the consent documents allowing their clinical information to be gathered and analyzed for research purpose. Subjects were divided into 3 categories: control group $(n=40)$, liver cirrhosis group without HCC (LC) who had CHC more than 6 months of infection $(n=$ 80) and HCC patients who had cirrhosis and were currently infected by HCV, but didn't start the treatments $(n=80)$. The staging of HCC patients was done using Okuda staging system respectively.

Venous blood samples $(10 \mathrm{~mL})$ were withdrawn from enrolled subjects by trained laboratory technicians. Each sample was divided into three portions: $4 \mathrm{ml}$ were collected in tubes containing EDTA for processing total RNA extraction, $4 \mathrm{~mL}$ were left to clot at room temperature, centrifuged and sera were separated for determination of biochemical parameters) and $2 \mathrm{ml}$ were collected in tubes containing EDTA for platelet count (PLT) by phoenix 3300 .

The following biochemical tests were done for all involved subjects: Aspartate aminotransferase (AST), alanine aminotransferase (ALT), alkaline phosphatase (ALP), total bilirubin (T. Bilirubin) and albumin were assayed using OLYMPUS automatic analyzer AU 400 using original reagents produced by Olympus Diagnostics GmbH (Irish Branch, Lismeehan, Ireland). Serum AFP level was determined using sandwich Enzyme Linked Immunosorbent Assay (ELISA) using washer (State fax (R) reader (state fax chromate-3033®) and kit for AFP (Pointe Scientific, Inc. 4559 Research drive, Canton MI 48188 USA).

\section{Determination of serum level of miR-155 and miR-665 by RT-qPCR}

Total RNA extraction and purification was done using a miRNeasy Mini Kit; cat no: 217004 (Qiagen, Hilden, Germany) according to the manufacturer's protocol. 
Reverse transcription: cDNA was synthesized by reverse transcription reaction using TaqMan MicroRNA Reverse Transcription Kit; cat no: 4366596 (Applied Biosystems, Foster city, USA) and the thermal cycler (Quanta Biotech).

Gene expression analysis: The quantification of miR155 and miR-665 levels was amplified from cDNA using TaqMan universal Master Mix and TaqMan assay (hasmiR-155; Catalog no: 4427975; Assay ID: 002623) and (hsa-miR-665; Catalog no: 4427975; Assay ID: 002681).The RNU6B snRNA was used as housekeeper gene (Catalog no: 4427975; Assay ID: 001093).

Mature miR-155 Sequence:

UUAAUGCUAAUCGUGAUAGGGGU

Mature miR-665 Sequence: ACCAGGAGGCUGAGGCCCCU

All samples were analyzed using the 5 plex Rotor-Gene PCR Analyzer (Qiagen, Germany). The $2^{\Delta \Delta C t}$ method was conducted for the analysis of gene expression levels using TaqMan microRNA Control Assays RNU6B as an endogenous reference control for normalization purposes. ${ }^{19}$

\section{Statistical analysis}

GraphPad Prism5® software (version 5.0a; GraphPad Software, Inc., San Diego, Calif) was used for the analysis of the data. Qualitative data were presented as frequencies $(\mathrm{n})$ and percentages $(\%)$. Chi-square $(\chi 2)$ test was used for comparisons between the three groups regarding the gender. Quantitative data were presented as mean \pm standard mean of error (SME). Comparison between the three groups used the one-way analysis of variance (ANOVA) test followed by Tukey test to compare the two groups. Comparison between three or more groups not normally distributed having quantitative variables used Kruskal-Wallis test (nonparametric test) followed by Mann-Whitney test to compare the two groups. Pearson's correlation coefficient was used to determine significant correlations between each miRNA-155 expression and miRNA-665 expression and other variables; the level of significant was $<0.05$. The diagnostic value was described by plotting sensitivity against specificity to obtain the receiver-operating characteristic (ROC) curve and both sensitivity and specificity were calculated.

\section{RESULTS}

The demographic features of the studied groups are shown in Table 1 . Both gender and age are matched among the three groups. Regarding the sex, there is a male predominance among HCC patients 64 males (80\%), which is matched in LC patients with 68 males $(85 \%)$ and controls group with 32 males (80\%).

There was a significant increase in the serum levels of AST, ALT, ALP, total bilirubin and AFP in HCC group in comparison with LC group. The serum levels of albumin and platelets count were significantly decreased in both LC group and HCC group as compared to the control group. In addition, the serum level of albumin and platelets count levels in the HCC patients were significantly lower than in the LC patients.

Tumor-related characteristics of HCC patients are shown at Table 2. Regarding Okuda staging, $31.3 \%$ of HCC patients were at stage I, $32.5 \%$ of them at stage II and $36.2 \%$ at stage III. Moreover, $43.7 \%$ of patients had single focal lesion and $56.3 \%$ of them had multiple (2-3) focal lesions. The focal lesion site was $42.5 \%$ in right lobe, $32.5 \%$ in left lobe and $25 \%$ in both left and right lobes. As for the tumor size, $53.7 \%$ of HCC patients were equal or less than $3.5 \mathrm{~cm}$ and $46.3 \%$ of them were more than $3.5 \mathrm{~cm}$.

As shown in Table 3, the mean serum level of miR-155 expression of both LC and HCC groups was significantly elevated to reach nearly (4-fold and 10-fold respectively) that of the control group. In addition, the mean serum level of miR-155 expression was significantly increased in the HCC group to reach nearly 3 -fold of the LC group.

On the other hand, regarding the mean serum level of miR-665 expression, a marked escalation in the HCC group that reached about 14-fold of both LC and control group.

As illustrated in Table 4, in the patients of LC, there was a significantly positive correlation between the mean serum level of miR-155 expression and the mean serum level of each of AST, ALT, ALP and AFP. On the other hand, there was a significantly negative correlation between the mean serum level of miR-155 expression and the mean serum level of PLT.

As shown in Table 4, in the patients of HCC, there was a significantly positive correlation between the mean serum level of miR-155 expression and the mean serum level of each one of the following: ALT, ALP and AFP.

Regarding the mean serum level of miR-665 expression, there was a significantly positive correlation between the mean serum level of miR-665 expression and each of the mean serum level of AST, ALT, ALP, AFP and tumor size.

On the other hand, there was a significantly negative correlation between the mean serum level of miR-665 expression and the mean serum level of Albumin.

Finally, there was a significantly positive correlation 
Mohamed et al.: MiR-155 and MiR-665 as a biomarkers for hepatocellular carcinoma for patients with chronic hepatitis $\mathrm{C}$ virus infection

\begin{tabular}{|c|c|c|c|c|}
\hline & $\begin{array}{l}\text { Control group } \\
(n=40)\end{array}$ & $\begin{array}{l}\text { LC group } \\
(n=80)\end{array}$ & $\begin{array}{l}\text { HCC group } \\
(n=80)\end{array}$ & (P-value) \\
\hline $\begin{array}{l}\text { Gender } \\
\text { Female } n(\%) \\
\text { Male } n(\%)\end{array}$ & $\begin{array}{l}8(20 \%) \\
32(80 \%)\end{array}$ & $\begin{array}{l}12(15 \%) \\
68(85 \%)\end{array}$ & $\begin{array}{l}16(20 \%) \\
64(80 \%)\end{array}$ & 0.670 \\
\hline $\begin{array}{l}\text { Age (years) } \\
\text { Mean } \pm \text { SEM }\end{array}$ & $50.75 \pm 1.275$ & $49.28 \pm 0.8622$ & $52.03 \pm 1.091$ & 0.1247 \\
\hline AST (IU/L) & $21.50 \pm 1.151$ & $75.39 \pm 2.297^{a}$ & $113.9 \pm 6.457^{a, b}$ & $<0.0001$ \\
\hline ALT (IU/L) & $13.85 \pm 0.5269$ & $56.38 \pm 3.154^{\mathrm{a}}$ & $108.9 \pm 5.519^{a, b}$ & $<0.0001$ \\
\hline ALP (IU/L) & $60.60 \pm 1.405$ & $99.21 \pm 3.106^{\mathrm{a}}$ & $250.7 \pm 10.73^{a, b}$ & $<0.0001$ \\
\hline Albumin $(\mathrm{g} / \mathrm{dL})$ & $4.435 \pm 0.08432$ & $2.846 \pm 0.09290^{\mathrm{a}}$ & $2.305 \pm 0.05676^{\mathrm{a}, \mathrm{b}}$ & $<0.0001$ \\
\hline T. Bilirubin (mg/dL) & $0.6550 \pm 0.03703$ & $1.246 \pm 0.04390^{\mathrm{a}}$ & $2.546 \pm 0.1368^{a, b}$ & $<0.0001$ \\
\hline $\operatorname{PLT}(\times 103 / \mu \mathrm{L})$ & $304.0 \pm 10.18$ & $192.9 \pm 4.307^{a}$ & $137.7 \pm 3.388^{a, b}$ & $<0.0001$ \\
\hline AFP (ng/mL) & $6.523 \pm 0.4850$ & $19.94 \pm 2.115^{\mathrm{a}}$ & $228.3 \pm 29.89^{a, b}$ & $<0.0001$ \\
\hline MiR-155 (RQ) & $1.277 \pm 0.1059$ & $4.561 \pm 0.4999^{a}$ & $13.20 \pm 1.587^{\mathrm{a}, \mathrm{b}}$ & $<0.0001$ \\
\hline MiR-665 (RO) & $1.232 \pm 0.1083$ & $1.286 \pm 0.1136$ & $17.37 \pm 1.138^{\mathrm{a}, \mathrm{b}}$ & $<0.0001$ \\
\hline
\end{tabular}

Values are expressed as Mean \pm standard error of mean (SEM).

AST: aspartate aminotransferase; ALT: alanine aminotransferase; ALP: alkaline phosphatase; T. Bilirubin: total bilirubin; PLT: platelets count; AFP: alpha fetoprotein.

a Significant difference from control group. ${ }^{\mathrm{b}}$ Significant difference from LC group.

\section{Table 2: Tumor-related characteristics of HCC patients}

Number of focal lesions

- Single $n(\%)$

- Multiple (2-3) $n(\%)$

Site of focal lesions:

- Rt. Lobe $n(\%)$

- Lt. Lobe $n(\%)$

- Both $n(\%)$

Tumor Size by CT:

- $\leq 3.5 \mathrm{~cm}, n(\%)$

- > $3.5 \mathrm{~cm}, n(\%)$

Okuda staging:

- Stage I

- Stage II

- Stage III

CT: Computed tomography.

between the mean serum level of both miR-155 and miR665 expression.

As shown in Figures 1-4, ROC analysis was conducted to identify the optimal levels of miR-155 and miR-665 expression for potential diagnostic value discriminating HCC among the high LC patients compared to the AFP level.

Alpha-fetoprotein best cut-off value was $>10.45$ with sensitivity of $57.5 \%$, specificity of $58.8 \%$, positive predictive value (PPV) of $58 \%$, negative predictive value (NPV) of $59 \%$ and accuracy of $58 \%$. On the other hand, miR-155 best cut-off value was $>4.30$ with sensitivity of
$35(43.7 \%)$

$45(56.3 \%)$

$34(42.5 \%)$

$26(32.5 \%)$

$20(25 \%)$

$43(53.7 \%)$

$37(46.3 \%)$

$25(31.3 \%)$

$26(32.5 \%)$

$29(36.2 \%)$ 


\begin{tabular}{|c|c|c|c|c|c|c|c|c|}
\hline & \multicolumn{4}{|c|}{ LC group } & \multicolumn{4}{|c|}{ HCC group } \\
\hline & \multicolumn{2}{|l|}{ MiR-155 } & \multicolumn{2}{|c|}{ MiR-665 } & \multicolumn{2}{|c|}{ MiR-155 } & \multicolumn{2}{|l|}{ MiR-665 } \\
\hline & $r$ & $P$-value & $r$ & $P$-value & $r$ & $P$-value & $r$ & $P$-value \\
\hline AST (IU/L) & 0.438 & $<0.0001$ & 0.014 & 0.903 & 0.216 & 0.054 & 0.392 & 0.0003 \\
\hline ALT (IU/L) & 0.272 & 0.015 & -0.168 & 0.137 & 0.282 & 0.011 & 0.475 & $<0.0001$ \\
\hline$A L P(I U / L)$ & 0.281 & 0.011 & 0.033 & 0.773 & 0.293 & 0.008 & 0.371 & 0.0007 \\
\hline Albumin $(g / d L)$ & -0.238 & 0.033 & -0.172 & 0.127 & -0.205 & 0.069 & -0.441 & $<0.0001$ \\
\hline T. Bilirubin (mg/dL) & -0.090 & 0.424 & -0.126 & 0.265 & 0.065 & 0.569 & 0.066 & 0.561 \\
\hline PLT $(\times 103 / \mu L)$ & -0.257 & 0.021 & -0.099 & 0.381 & -0.048 & 0.674 & -0.155 & 0.170 \\
\hline AFP (ng/mL) & 0.378 & 0.0006 & 0.179 & $0.112-$ & 0.315 & 0.004 & 0.518 & $<0.0001$ \\
\hline Tumor size $(\mathrm{cm})$ & - & - & - & & 0.063 & 0.577 & 0.533 & $<0.0001$ \\
\hline MiR-155 (RQ) & - & - & 0.172 & 0.126 & - & - & 0.423 & $<0.0001$ \\
\hline MiR-665 (RQ) & 0.172 & 0.126 & - & - & 0.423 & $<0.0001$ & - & - \\
\hline
\end{tabular}

AST: aspartate aminotransferase; ALT: alanine aminotransferase; T. Bilirubin: total bilirubin; PLT: platelets Count; AFP: alpha fetoprotein

\begin{tabular}{lllllll}
\hline \multicolumn{7}{l}{ Table 4: Diagnostic performance of AFP, miR-155, miR-665 for discriminating HCC patients from LC patients } \\
\hline & Cut-off & Sensitivity \% & Specificity \% & AUC & P-value & 95\% Confidence Interval \\
\hline AFP(ng/mL) & $>10.45$ & 57.5 & 58.8 & 0.608 & 0.18 & 0.507 to 0.710 \\
MiR-155 (RQ) & $>4.30$ & 80 & 62.5 & 0.743 & $<0.0001$ & 0.666 to 0.820 \\
MiR-665 (RQ) & $>2.23$ & 92.5 & 86.3 & 0.930 & $<0.0001$ & 0.879 to 0.980 \\
\hline
\end{tabular}

In our investigation, HCC patients didn't include alcoholic liver disease, nonalcoholic fatty liver disease nor autoimmune liver disease. Our results showed that the mean serum level of miR-155 expression was significantly higher in both LC and HCC patients compared to the controls. In addition, the mean serum level of miR-155 expression was significantly elevated in the HCC group in comparison with that of the LC group.

Our finding came in agreement with Ezzat et al., 2016 ${ }^{[20]}$ whose work found that there was an overexpression of miR-155 in the serum of both LC and HCC patients in comparison with the controls.

Also, our results were in accordance with Guan et al., 2016 ${ }^{[21]}$ who measured the level of miR-155 expression in HCC tissue against the neighboring free cancer cells and declared its elevation in the cancer cells.

Generally, these findings further support the idea that miR-155 forms an important bridge between inflammation represented by $\mathrm{CHC}$ patients with cirrhosis group and tumorigenesis represented by HCC group indicating that
miR-155 has an important role in the diagnosis of HCC in LC patients previously infected by HCV.

There is a direct positive correlation between miR-155 and TNF- $\alpha$ where both factors are considered a positive regulator for the other. ${ }^{[2]} \mathrm{TNF}-\alpha$ is an important cytokine in the inflammation process and regulated by the nuclear factor kappa-light-chain-enhancer of activated B cells (NF- $x \mathrm{~B})$ transcription factor at the level of transcription. $\mathrm{NF}-x \mathrm{~B}$ pathway is induced by some ligands and receptors as Toll-like receptors (TLRs). ${ }^{[23]}$ Regarding the HCV infection, hepatitis $\mathrm{C}$ virus core protein and Non-structural protein 3 causes a direct increase in the production of TNF- $\alpha$ in B cells. In addition, HCV induces the NF- $x \mathrm{~B}$ pathway by interacting with TLR 8 and 4 where HCV as a single stranded RNA virus interacts with TLR 8 and nonstructural protein 5 interacts with TLR 4 in Kupffer cells. ${ }^{[2]}$

Regarding the elevation of miR-155 in HCC patients, a possible explanation for these results may be presented by Li et al., 2018, ${ }^{[24]}$ who showed that stimulation of Tumor Growth Factor- $\beta 1$ results in the raise of miR-155 expression in HCC cells and this escalation of miR-155 
promotes epithelial-mesenchymal transition (EMT), invasion and migration of HCC cells.

In addition, MiR-155 targets adenomatous polyposis coli (APC) gene, which is a negative regulator of Wnt signaling, which is an important signaling factor implicated in the control of cell apoptosis and proliferation and is reported to be one of the main cascades that regulates cancer development. ${ }^{[25-27]}$

Moreover, miR-155 has a dual action in case of cancer - it may act as onco-suppressor or oncogene, which is the case in hepatocellular carcinoma. ${ }^{[28]}$

In HCC, miR-155 acts as an oncogene resulting in tumor progression by the inhibition of multiple genes that act as tumor suppressors and this offers a third mechanism or for the escalation of serum miR-155 expression level in HCC patients.

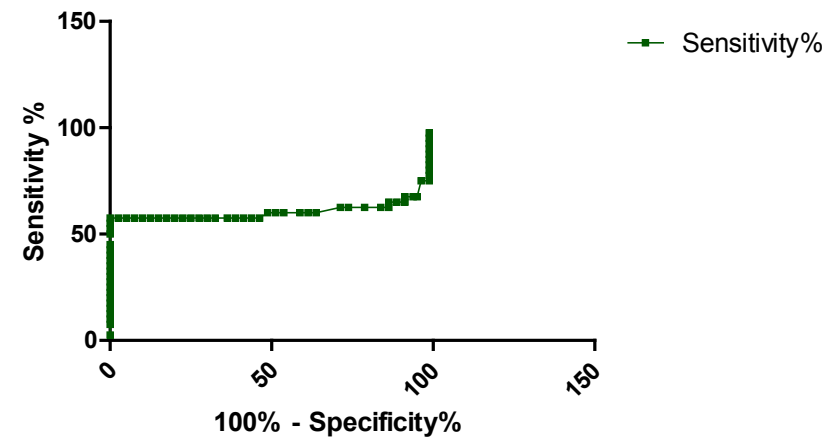

Figure 1: ROC curve of AFP in discriminating HCC group from LC group

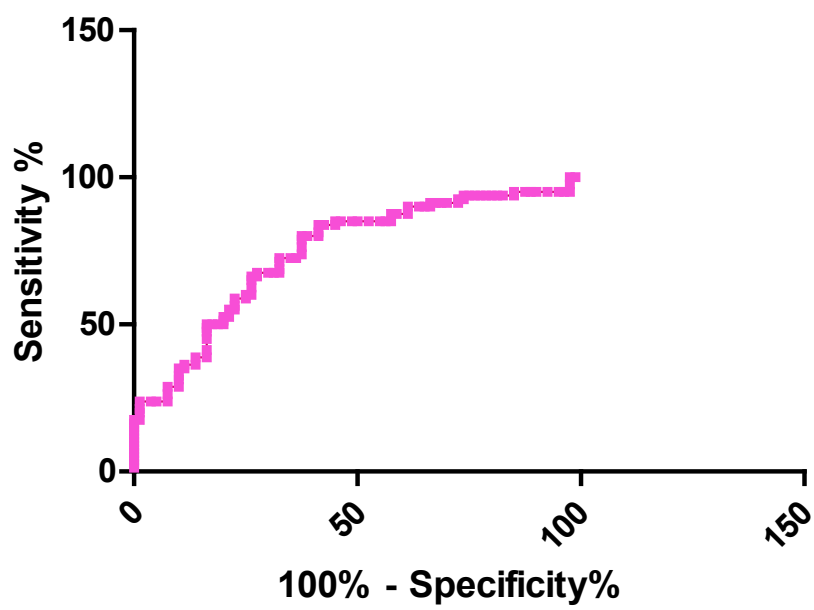

Figure 2: $R O C$ curve of $R O$ of miR-155 in discriminating HCC group from LC group
Cytosine-cytosine-adenosine-adenosine-thymidineenhancer-bindingproteins (CCAATor C/EBPs) was often increased in HCC; hence, it was assessed as the first tumor suppressor that high level of miR-155 resulted in its knock down. ${ }^{[29]}$

In addition, sex-determining region Y-gene related high-mobility-group box gene (SOX6) is another tumor suppressor that was suppressed due to the increase in miR-155 expression level. The inhibition of SOX6 leads to the inactivation of $\mathrm{P} 21$ growth regulator promoting HCC development. ${ }^{[30]}$

Moreover, suppressor cytokine signaling 1 (SOCS1) is a tumor suppressor that is also considered as a target for miR-155. Downregulation of SOCS1 by miR-155 elevation causes the activation of Signal transducers and activators of transcription 3 (STAT3) that inhibits apoptosis in cancer cells including HCC. ${ }^{[31,32]}$

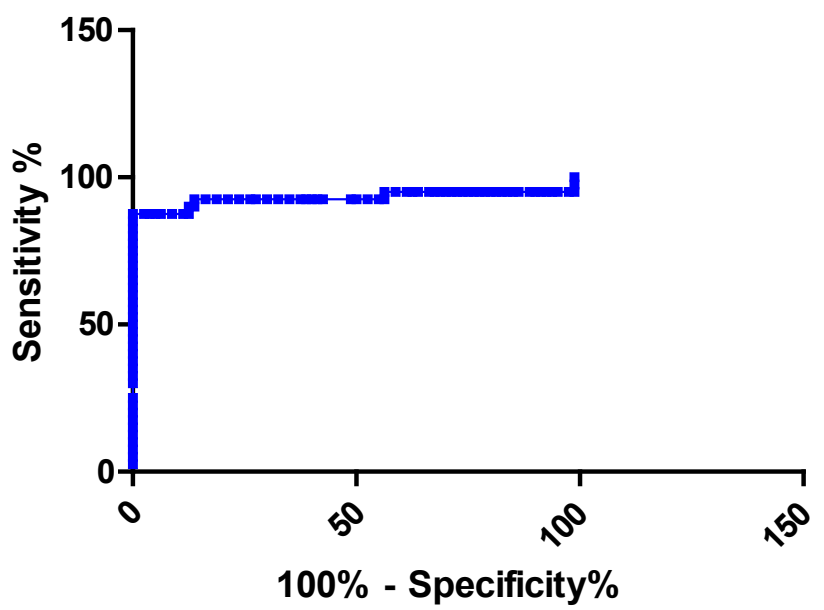

Figure 3: ROC curve of RQ of miR-665 in discriminating HCC group from LC group

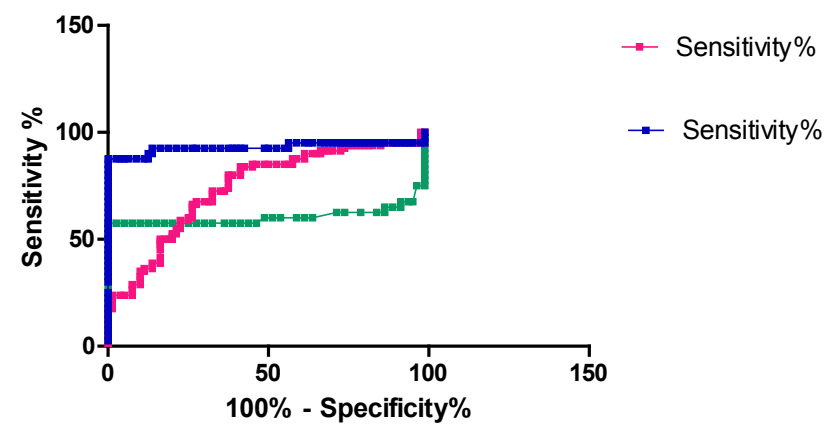

Figure 4: Combined ROC curves of $R 0$ of miR-665, RO of miR-155 and AFP in discriminating HCC group from LC group 
Furthermore, the fourth tumor suppressor that is inhibited by miR-155 uprising is called F-box/WD repeat-containing protein 7 (FBXW7). FBXW7 is believed to be the center of multiple pathways including controlling cell growth, cell differentiation and tumor genesis. ${ }^{[3,34]}$

Finally, another tumor suppressor that was examined as destination for miR-155 was suppressing Phosphatase and Tensin homolog (PTEN) through inhibiting the action of class I phosphatidylinositol 3-kinases (PI3K)/ Akt pathway. ${ }^{[18]}$

Regarding miR-665 expression level, it was increased in some tumors as intestinal gastric adenocarcinoma ${ }^{[35]}$ but decreased in others as osteosarcoma. ${ }^{[36]}$ This may suggest that miR665 exerts a different biological function according to the type of tumors. As for the expression of miR-665 in HCC, previous studies reported that there was an increase in the serum exosomal expression of miR-665 in HCC patients in comparison to people in good health. ${ }^{[37,38]}$

The current study showed that the serum level of miR665 expression in the HCC group was markedly higher than both LC and control groups. However, there was no significant difference between the LC patients and the control group.

Another study by Qu et al., 2017 ${ }^{[39]}$ demonstrated that serum level of exosomal miR-665 in HCC group of patients was significantly upregulated when measured against a healthy group of people.

In addition, Hu et al., 2018 $8^{[16]}$ worked on HCC tissue cells and illustrated that there was a significant elevation of miR-665 expression in the HCC cells when compared to the surrounding non-cancerous cells.

However, our results showed that there was a significant increase of miR-665 expression in the free serum of HCC patients in comparison with the control group, which gives the benefit of removing the step of differential centrifugation and density-gradient centrifugation required for serum exosomal extraction.

The increase in miR-665 expression in HCC could be explained by Qu et al., 2017 ${ }^{[38]}$ who showed that exosomes extracted from hepatocellular tumor cells can use its content of miR-665 to activate the MAPK/ERK pathway. The activation of the MAPK/ERK pathway through miR665 is considered an important intracellular proliferative and anti-apoptotic pathway and takes part in the malignant proliferation of tumor cells by affecting the activity of effector molecules, such as downstream cell cycle regulatory proteins and apoptosis-related proteins. ${ }^{[39]}$
In addition, another possible explanation for the elevation of miR-665 expression in HCC was introduced by $\mathrm{Hu}$ et al., $2018^{[16]}$ who illustrated that miR-665 is targeting a tumor suppressor called protein tyrosine phosphatase receptor type $\mathrm{B}$ (PTPRB). This gene can modulate Hippo signaling activity, which is a central mechanism that suppresses the overgrowth of the tissue leading to the inhibition of carcinogenesis. Based on these reasons, miR-665 promotes hepatic cancer proliferation, migration, and invasion by inhibition of Hippo pathway activity through PTPRB.

In the current study and in the matter of the correlation between the two biomarkers and the other variables in patients of LC, there was a significantly positive correlation between the mean serum level of miR-155 expression and the mean serum level of each of AST, ALT, ALP and AFP. On the other hand, there was a significantly negative correlation between the mean serum level of miR-155 expression and the mean serum level of PLT.

Our data was consistent with RIAD et al., 2015, ${ }^{[40]}$ who reported a positive correlation between the serum level of miR-155 and both ALT and AST.

Also, a previous study by Kałużna, $2014{ }^{[17]}$ showed that hepatic expression of miRNA-155 correlates with the serum level of biochemical markers of liver damage.

In the present study and in the matter of the correlation between the two biomarkers and the other variables in patients of HCC, there was a significantly positive correlation between the mean serum level of miR-155 expression and the mean serum level of each of ALT, ALP and AFP.

Regarding the mean serum level of miR-665 expression, there was a significantly positive correlation between the mean serum level of miR-665 expression and each of the mean serum levels of AST, ALT, ALP, AFP and tumor size.

On the other hand, there was a significantly negative correlation between the mean serum levels of miR-665 expression and the mean serum level of Albumin.

Finally, there was a significantly positive correlation between the mean serum level of both miR-155 and miR665 expression.

Our results agreed with Guan et al., 2016, ${ }^{[21]}$ who revealed the absence of association between miR-155 expression and tumor size in HCC patients however, in contrast to our results, they showed no association between miR-155 expression and AFP level. 
In addition, our results came in agreement with Ezzat et al., 2016, ${ }^{[20]}$ who found that was no significant impact of tumor characteristics regarding number; site; size and serum values of miRNA-155.

Moreover, our results were in consistence with $\mathrm{Qu}$ et al., $2017,{ }^{[38]}$ who reported that a high-expression of miR-665 was found in the higher clinical stages patients of HCC; also a high-expression of miR-665 was associated with larger tumor size.

The most used marker in diagnosis of HCC is the AFP serum level in spite of its humble sensitivity $(39 \%-65 \%) .{ }^{[8]}$

Regarding the discrimination of HCC patients from the high risk LC patients, both miR-155 and miR-665 had the ability to differentiate between the two groups with sensitivity $80 \%$, specificity $62.5 \%$, PPV 79\%, NPV 63\% and accuracy $71 \%$. and sensitivity $90 \%$, specificity $95 \%$, PPV 93\%, NPV \% 86\% and accuracy 89\% respectively in comparison with AFP with sensitivity $60 \%$, specificity $70 \%$, PPV 58\%, NPV 59\% and accuracy 58\%.

According to the previous results, miR-155 didn't show a better ability in the diagnosis with specificity of only $62.5 \%$ than the common marker AFP, while miR-665 approved to be a good diagnostic biomarker with good sensitivity, specificity, PPV, NPV and diagnostic accuracy.

Another study by Ezzat et al., $2016^{[20]}$ reported that in the discrimination of HCC patients from CHC patients, miR155 serum expression had $95 \%$ sensitivity, $76.5 \%$ specificity compared to the AFP serum level with $100 \%$ sensitivity, $69.2 \%$ specificity.

\section{STUDY LIMITATION}

Hepatocellular carcinoma group didn't include patients with alcoholic liver disease, nonalcoholic fatty liver disease and autoimmune liver disease, and we recommend future involvement of this condition.

\section{CONCLUSION}

The following conclusions can be drawn from the present study:

Serum miRNA-665 is a good noninvasive diagnostic biomarker for detection of HCC in patients with LC as compared to AFP serum level as a reference.

Serum miR-155 didn't show a better diagnostic ability than serum AFP due to relative low specificity that makes it hard to be used in clinical practice in differentiation between HCC patients and high-risk LC patients.
The positive correlation between serum miR-665 and the tumor size of HCC is suggesting a promising role of miR665 in the prognosis of the disease.

\section{Conflict of Interest}

The authors declare no conflicts of interests.

\section{REFERENCES}

1. Axley P, Ahmed Z, Ravi S, Singal AK. Hepatitis C Virus and Hepatocellular Carcinoma: A Narrative Review. J Clin Transl Hepatol 2018;6:1-6.

2. Kamal SM. Hepatitis $C$ virus genotype 4 therapy: Progress and challenges. Liver Int 2011;31:45-52.

3. Torre LA, Bray F, Siegel RL, Ferlay J, Lortet-Tieulent J, Jemal A. Global cancer statistics, 2012. CA Cancer J Clin 2015;65:87-108.

4. El-Serag HB. Epidemiology of Viral Hepatitis and Hepatocellular Carcinoma. Gastroenterology 2012;142:1264-73.

5. Biondi A, Malaguarnera G, Vacante M, Berretta M, D'Agata V, Malaguarnera $\mathrm{M}$, et al. Elevated serum levels of Chromogranin A in hepatocellular carcinoma. BMC Surg 2012;12:S7.

6. Qi J, Wang J, Katayama H, Sen S, Liu SM. Circulating microRNAs (cmiRNAs) as novel potential biomarkers for hepatocellular carcinoma. Neoplasma 2013;60:135-42.

7. Huang JT, Liu SM, Ma H, Yang Y, Zhang X, Sun H, et al. Systematic Review and Meta-Analysis: Circulating miRNAs for Diagnosis of Hepatocellular Carcinoma. J Cell Physiol 2016;231:328-35.

8. Berretta M, Cavaliere C, Alessandrini L, Stanzione B, Facchini G, Balestreri L, et al. Serum and tissue markers in hepatocellular carcinoma and cholangiocarcinoma: clinical and prognostic implications. Oncotarget 2017;8:14192-220.

9. Stefaniuk P, Cianciara J, Wiercinska-Drapalo A. Present and future possibilities for early diagnosis of hepatocellular carcinoma. World J Gastroenterol 2010;16:418-24.

10. O'Connell RM, Rao DS, Chaudhuri AA, Baltimore D. Physiological and pathological roles for microRNAs in the immune system. Nat Rev Immunol. 2010;10:111-22.

11. Witwer KW. Circulating MicroRNA biomarker studies: Pitfalls and potential solutions. Clin Chem 2015;61:56-63.

12. Erener S, Mojibian M, Fox JK, Denroche HC, Kieffer TJ. Circulating miR375 as a biomarker of $\beta$-cell death and diabetes in mice. Endocrinology 2013;154:603-8.

13. Vickers KC, Palmisano BT, Shoucri BM, Shamburek RD, Remaley AT. MicroRNAs are transported in plasma and delivered to recipient cells by high-density lipoproteins. Nat Cell Biol 2011;13:423-33.

14. Valadi H, Ekström K, Bossios A, Sjöstrand M, Lee JJ, Lötvall JO. Exosomemediated transfer of mRNAs and microRNAs is a novel mechanism of genetic exchange between cells. Nat Cell Biol 2007;9:654-9.

15. Iorio M V, Croce CM. MicroRNAs in cancer: Small molecules with a huge impact. J Clin Oncol 2009;27:5848-56.

16. Hu Y, Yang C, Yang S, Cheng F, Rao J, Wang X. miR-665 promotes hepatocellular carcinoma cell migration, invasion, and proliferation by decreasing Hippo signaling through targeting PTPRB. Cell Death Dis 2018;9:954

17. Kałużna EM. MicroRNA-155 and microRNA-196b: promising biomarkers in hepatitis C virus infection? Rev Med Virol 2014;24:169-85.

18. Fu X, Wen H, Jing L, Yang Y, Wang W, Liang X, et al. MicroRNA-155-5p promotes hepatocellular carcinoma progression by suppressing PTEN through the PI3K/Akt pathway. Cancer Sci 2017;108:620-31.

19. Chen C, Tan R, Wong L, Fekete R, Halsey J. Quantitation of MicroRNAs by Real-Time RT-qPCR. Methods Mol Biol 2011,687:113-34.

20. Ezzat WM, Amr KS, Raouf HA, Elhosary YA, Hegazy AE, Fahim HH, et al. Relationship Between Serum microRNA155 and Telomerase Expres- 
sion in Hepatocellular Carcinoma. Arch Med Res 2016;47:349-55.

21. Guan C, Yang F, He X, Li T, Yang Q, He H, et al. Clinical significance of microRNA-155 expression in hepatocellular carcinoma. Oncol Lett 2016;11:1574-80.

22. Bala S1, Tilahun Y, Taha O, Alao H, Kodys K, Catalano D, et al. Increased microRNA-155 expression in the serum and peripheral monocytes in chronic HCV infection. J Transl Med 2012;10:151.

23. Hayden MS, Ghosh S. Regulation of NF- $\kappa B$ by TNF family cytokines. Semin Immunol 2014;26:253-66.

24. Li DP, Fan J, Wu YJ, Xie YF, Zha JM, Zhou XM. MiR-155 up-regulated by TGF- $\beta$ promotes epithelial-mesenchymal transition, invasion and metastasis of human hepatocellular carcinoma cells in vitro. Am J Transl Res 2017;9:2956-65.

25. Sato N, Yamabuki T, Takano A, Koinuma J, Aragaki M, Masuda K, et al. Wnt Inhibitor Dickkopf-1 as a Target for Passive Cancer Immunotherapy. Cancer Res 2010;70:5326-36.

26. Zhang $\mathrm{Y}$, Wei $\mathrm{W}$, Cheng $\mathrm{N}$, Wang $\mathrm{K}, \mathrm{Li} \mathrm{B}$, Jiang $\mathrm{X}$, et al. Hepatitis $\mathrm{C}$ virusinduced up-regulation of microRNA-155 promotes hepatocarcinogenesis by activating Wnt signaling. Hepatology 2012;56:1631-40.

27. Zhan T, Rindtorff N, Boutros M. Wnt signaling in cancer. Oncogene 2017;36:1461-73.

28. Chen Z, Ma T, Huang C, Hu T, Li J. The pivotal role of microRNA-155 in the control of cancer. J Cell Physiol 2014;229:545-50.

29. Wang B1, Majumder S, Nuovo G, Kutay H, Volinia S, Patel T, et al. Role of microRNA-155 at early stages of hepatocarcinogenesis induced by choline-deficient and amino acid-defined diet in C57BL/6 mice. Hepatology 2009;50:1152-61.

30. Xie Q, Chen X, Lu F, Zhang T, Hao M, Wang Y, et al. Aberrant expression of microRNA 155 may accelerate cell proliferation by targeting sex-determining region y box 6 in hepatocellular carcinoma. Cancer 2012;118:2431-42.

31. Siveen KS, Sikka S, Surana R, Dai X, Zhang J, Kumar AP, et al. Targeting the STAT3 signaling pathway in cancer: Role of synthetic and natural inhibitors. Biochim Biophys Acta 2014;1845:136-54.
32. Yan XL, Jia YL, Chen L, Zeng Q, Zhou JN, Fu CJ, et al. Hepatocellular carcinoma-associated mesenchymal stem cells promote hepatocarcinoma progression: Role of the S100A4-miR155-SOCS1-MMP9 axis. Hepatology 2013;57:2274-86.

33. Roversi G, Picinelli C, Bestetti I, Crippa M, Perotti D, Ciceri S, et al. Constitutional de novo deletion of the FBXW7 gene in a patient with focal segmental glomerulosclerosis and multiple primitive tumors. Sci Rep 2015;5:15454.

34. Tang B, Lei B, Qi G, Liang X, Tang F, Yuan S, et al. MicroRNA-155-3p promotes hepatocellular carcinoma formation by suppressing FBXW7 expression. J Exp Clin Cancer Res 2016;35: 93.

35. Chen J, Sun D, Chu H, Gong Z, Zhang C, Gong B, et al. Screening of differential microRNA expression in gastric signet ring cell carcinoma and gastric adenocarcinoma and target gene prediction. Oncol Rep 2015;33:2963-71.

36. Dong C, Du Q, Wang Z, Wang Y, Wu S, Wang A. MicroRNA-665 suppressed the invasion and metastasis of osteosarcoma by directly inhibiting RAB23. Am J Transl Res 2016;8:4975-81.

37. Li S, Yao J, Xie M, Liu Y, Zheng M. Exosomal miRNAs in hepatocellular carcinoma development and clinical responses. J Hematol Oncol 2018;11:54.

38. Qu Z, Wu J, Wu J, Ji A, Qiang G, Jiang Y, et al. Exosomal miR-665 as a novel minimally invasive biomarker for hepatocellular carcinoma diagnosis and prognosis. Oncotarget 2017;8:80666-78.

39. Carlo-Stella C, Locatelli SL, Giacomini A, Cleris L, Saba E, Righi M, et al. Sorafenib Inhibits Lymphoma Xenografts by Targeting MAPK/ERK and AKT Pathways in Tumor and Vascular Cells. PLoS One 2013;8: e61603.

40. Riad SE, El-Ekiaby N, Mekky RY, Ahmed R, El Din MA, El-Sayed M, et al. Expression signature of microRNA-155 in hepatitis $C$ virus genotype 4 infection. Biomed Reports 2015;3:93-7.

How to cite this article: Mohamed AA, Ali Omar AA, EL-Awady RR, Aboelsayed Hassan SM, Soliman Eitah WM, Ahmed R, et al. MiR-155 and MiR-665 role as potential non-invasive biomarkers for hepatocellular carcinoma in Egyptian patients with chronic hepatitis C virus infection. J TransI Int Med 2020; 8: 32-40. 